\title{
Vocalizations by a Sexually Dimorphic Isolated Larynx: Peripheral Constraints on Behavioral Expression
}

\author{
Martha L. Tobias and Darcy B. Kelley \\ Department of Biological Sciences, Columbia University, New York, New York, 10027
}

The clawed frog Xenopus laevis uses sexually dimorphic vocalizations, mate calling and ticking, to advertise reproductive state. The basic unit of vocalization is a brief click, produced by the movement of cartilagenous disks located within the larynx. The rate of click production in the malespecific mate call $(71 \mathrm{~Hz})$ is an order of magnitude faster than the rate of click production in female typical ticking (6 $\mathrm{Hz}$ ). To determine if vocalization rate is constrained by the periphery, male and female larynges were isolated and response of the muscles to nerve stimulation was studied. Laryngeal muscle response is markedly dimorphic in the 2 sexes, both in the amplitude potentiation of electromyograms and in the rate at which discrete tension transients can be produced. At $6 \mathrm{~Hz}$ (ticking), both sexes generate discrete tension transients in response to each stimulus pulse. In response to nerve stimulation at $71 \mathrm{~Hz}$ (mate calling), male laryngeal muscle generates discrete tension transients while female laryngeal muscle does not.

Since expression of sex-specific vocalizations is regulated by androgenic hormones, responses of laryngeal muscle to nerve stimulation in androgen-treated adult females and castrated adult males were also examined. The responses of laryngeal muscle from castrated and intact males are similar. Androgen-treated female larynx is partially masculinized but does not produce tension transients at the mate call rate. These physiological results are in close agreement with behavioral observations.

Sounds produced by the isolated larynx were nearly identical in spectral properties to those produced by an intact male. We determined that the production of a discrete tension transient is prerequisite to click production. Thus, one reason females do not mate call, even when treated with androgens, is that female laryngeal muscle cannot produce discrete tension transients at a rapid rate.

The release of steroid hormones at critical periods during development controls the ultimate expression of many sexually dimorphic behaviors in adults (Goy and McEwen, 1980). Typically, these behaviors contribute to the reproductive success of the animal. Both neural and muscular elements act in concert

Received Oct. 10, 1986; revised Mar. 2, 1987; accepted Mar. 4, 1987.

We are grateful to Michael Scudder, David Sassoon, Eduardo Macagno, and Martin Chalfie for critical review of the manuscript. This work was supported by 5R1NS23684-05 (to D.B.K.) and 5F32NS07685-02 (to M.L.T.).

Correspondence should be addressed to Martha L. Tobias, Columbia University, Department of Biological Sciences, 909 Sherman Fairchild, New York, NY 10027.

Copyright (C) 1987 Society for Neuroscience $0270-6474 / 87 / 103191-07 \$ 02.00 / 0$ to produce the behavior. An unresolved issue is whether the locus of regulation for a particular aspect of behavior can be confined to the CNS or the periphery. Identifying such regulatory points is a critical first step in determining how hormone action controls sexually dimorphic behaviors. To explore this question, sexually dimorphic vocalizations in the frog, Xenopus laevis, were studied. Specifically, the ability of the periphery to restrict the rate of vocal production was determined. The effector organ for vocalization, the larynx, was isolated and responses of the muscle to nerve stimulation were examined. Laryngeal muscle responses were compared in adult male, female, castrated male, and testosterone-treated female animals. The ability of the larynx to produce sounds in vitro allowed us to identify those properties responsible for the observed behavioral dimorphism.

In $X$. laevis, expression of the male-specific vocalization, the mate call, is under strict androgen regulation. Males mate-call, females do not. Castrated males cease calling within 3 weeks; however, mate calling is reinstated following treatment with androgens (Wetzel and Kelley, 1983). These results suggest that females might not mate-call because of a lack of sufficient circulating androgen. However, androgen treatment of adult females does not enable them to mate-call (Hannigan and Kelley, 1986). Both the neurons of the CNS vocal pathway and the laryngeal muscles have androgen receptors (Kelley et al., 1975; Kelley, 1981; Segil et al., 1987). The inability of androgen to masculinize vocal behaviors in adult females could be due either to the inability of the CNS to generate the mate-call pattern or to the inability of laryngeal muscles to respond to this activity.

The vocalizations of $X$. laevis are composed of trains of clicks (see Kelley, 1986, for review) produced by movement of opposed disks of arytenoid cartilage located at the anterior pole of the larynx (Ridewood, 1898; Yaeger, 1982). The dilator laryngis or bipinnate muscles that flank the laryngeal skeleton insert onto these disks via a tendinous sheath and are responsible for their movement. The bipinnate muscles are innervated, at their caudal pole, by axons of motor neurons in cranial nucleus IX-X (Kelley, 1981) that exit the medulla in the most cauda nerve rootlet of the cranial nerve IX-X complex (Simpson et al., 1986). The anatomical connections of these laryngeal motor neurons have been mapped (Wetzel et al., 1985) and correspond to CNS nuclei demonstrated to participate in the production of vocalizations in other anurans (Schmidt, 1976, 1983, 1984).

The male specific mate call is used to attract and excite females (Picker, 1983). The female typical call, ticking, is used by sexually unreceptive females to terminate clasping by males (Kelley, 1982; Weintraub et al., 1985). Male and female vocalizations are dimorphic with respect to the rate and temporal pattern 
Electromyogram and tension records from male and female isolated larynges. The rate of stimulation is indicated at left. Top trace, Stimulus monitor; middle trace, compound action potential records from the bipinnate muscle; bottom trace, isotonic tension records from the bipinnate muscle. Oscillations between tension transients in $A$ and following the stimulus train in $C$ result from passive responses of the suture thread attaching the tendon to the transducer following rapid release of tension. Calibration bar: vertical, compound action potential $-5 \mathrm{mV}(A$, $C), 20 \mathrm{mV}(B, D)$, tension $=3.5 \mathrm{gm}$; horizontal, $20 \mathrm{msec}$.
A

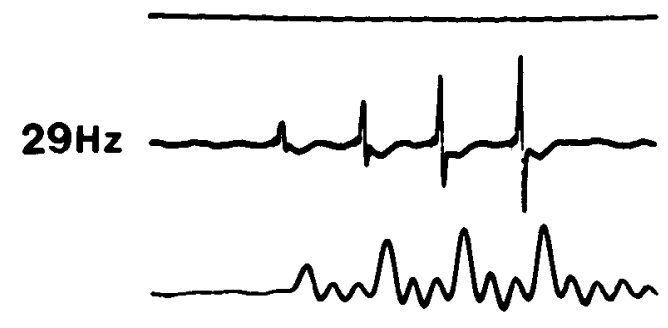

ठ́

C

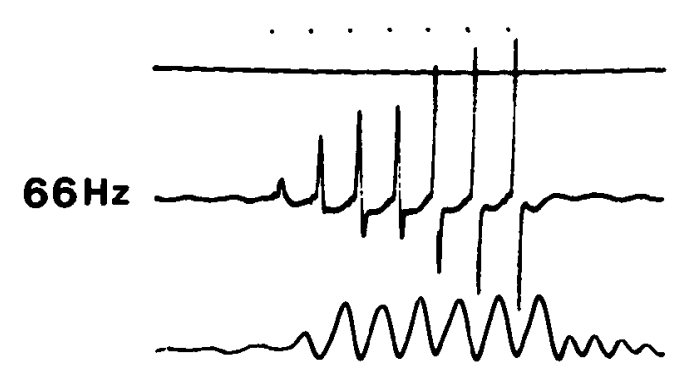

B $\quad$ q
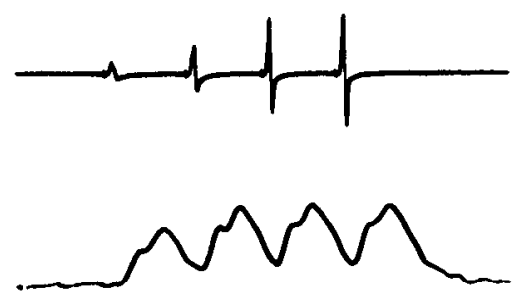

D

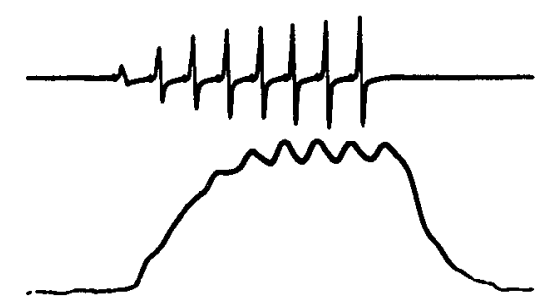

of clicks. The mate call is composed of alternating fast $(71 \mathrm{~Hz}$, $250 \mathrm{msec}$ duration) and slow ( $36 \mathrm{~Hz}, 750 \mathrm{msec}$ duration) trills (Wetzel and Kelley, 1983), while ticking is composed of one uniform slow rate of $6 \mathrm{~Hz}$ (Hannigan and Kelley, 1986). Since vocalizations are highly stereotyped, easily distinguished between the 2 sexes, and under strict hormonal regulation, they provide an ideal system with which to ask questions about the locus of action of androgenic hormones.

\section{Materials and Methods}

Thirty-one sexually mature frogs ( 10 male, 21 female) were purchased from Nasco (Fort Atkinson, WI) or Xenopus I (Ann Arbor, MI). Animals were maintained on a 12:12 light : dark cycle, in plastic tanks, and fed frog brittle 3 times a week. Seven intact male and 8 intact female frogs were tested. Thirteen female frogs were treated with androgenic steroids for 2-4 weeks. Six of these were ovariectomized (Kelley et al., 1975) and received a $20 \mathrm{mg}$ pellet implant of testosterone propionate into the dorsal lymph sac. This dose had previously been shown to alter vocalizations in females (Hannigan and Kelley, 1986) and to increase muscle SDHase activity (Gray et al., 1985). Circulating androgen levels produced by this dose exceed circulating titers in intact males (Kelley, 1980). The remaining 7 females received implants but were not gonadectomized. Three castrated males were also studied. Circulating androgen titers in castrated males are below detectable levels (less than $7 \mathrm{pg}$ ) $\mathrm{ml}$; T. Lambdin and D. B. Kelley, unpublished observations).

Preparation of isolated larynx. Animals were anesthetized by immersion in MS 222 ( $\mathrm{m}$-amino benzoate, methane sulfonate, Aldrich Chemical Co., 1\%), and placed ventral side up, and the skin and musculature of the abdominal region were removed. The heart, situated just ventral to the laryn $x$, was excised and the larynx gently lifted, severing all attachments to the body. The larynx was placed, dorsal side up, in a wax-coated $35 \mathrm{~mm}$ Petri dish, pinned in place, and bathed in glucosesupplemented frog Ringer's solution $(\mathrm{NaCl}, 0.1 \mathrm{~m} ; \mathrm{KCl}, 2 \mathrm{mM} ; \mathrm{CaCl}$, $2.5 \mathrm{~mm}$; MgCl, $3.0 \mathrm{~mm}$; HEPES, $4 \mathrm{~mm}$; glucose, $27.7 \mathrm{~mm}$ ). Cartilage, connective tissue, and fat were carefully removed to reveal the laryngeal muscles and nerve.

Recording and stimulating. The laryngeal nerve was drawn into a suction electrode and stimulated (WPI 302-T) with suprathreshold trains at varying rates. The stimulus train was $250 \mathrm{msec}$, which is equal to the fast portion of the mate call in intact males. Bipolar silver electrodes (36 gauge), insulated to the tip, were used to record electromyograms. A force transducer (Grass, FT-103) connected to the tendon of the bipinnate muscle, just before its insertion into the arytenoid disk, was used to measure isotonic tension of the muscle. Tension records were amplified (Grass, PC 16) and displayed on a storage oscilloscope. All data were photographed directly from the oscilloscope (Tektronix polaroid camera, C-59A).

In some experiments, sounds produced by the larynx were recorded by placing a small condenser microphone (Knowles, XL-9073) close to the arytenoid disks. The signal was amplified and recorded (Akai GX255). Sounds were subsequently analyzed with a sonograph (Kay 7800).

Data analysis. The height of the first compound action potential given in response to a stimulus train $(a)$ and the height of the largest compound action potential in the train $(b)$ were measured using a Summagraphics bit pad. The potentiation index (PI) was then computed as $b / a$.

Transient tension $(T t)$ was defined as the tension produced in response to an individual stimulus pulse. Tension records that rise and fall back to baseline in response to an individual stimulus pulse are referred to as discrete tension transients. A maintained tension $(T M)$ does not fluctuate between stimulus pulses but is sustained throughout the stimulus train. The amplitude of discrete tension records was measured as the height from the apex of the record to baseline. Transients superimposed on a maintained tension were measured from the apex of the transient to the apex of the maintained tension. The percentage transient tension was computed as $T t /(T t+T m) \times 100$. Statistical significance of differences between males and females and between testosteronetreated and control females for the PI and the percentage transient tension was determined using the Wilcoxon rank sign test (Snedecor and Cochran, 1967).

\section{Results}

Sexual dimorphism in physiological properties of the larynx Two physiological properties of the isolated larynx were measured in response to stimulation of the laryngeal nerve: compound action potential amplitude and isotonic tension produced by the bipinnate muscle. The primary difference between female and male responses is the rate at which females can produce discrete tension transients. Figure 1 compares responses from 


\begin{tabular}{|c|c|c|c|c|c|c|c|}
\hline \multicolumn{2}{|l|}{ Male } & \multicolumn{2}{|c|}{ Female } & \multicolumn{2}{|c|}{ T-female } & \multicolumn{2}{|c|}{ Castrated male } \\
\hline$\underline{\text { PI }}$ & $\% \mathrm{Tt}$ & PI & $\% \mathrm{Tt}$ & PI & $\% \mathrm{Tt}$ & PI & $\% \mathrm{Tt}$ \\
\hline 3.8 & 100 & 1.3 & 18 & 1.3 & 20 & $4.0^{6}$ & 100 \\
\hline 5.6 & 100 & 1.5 & 27 & $2.2^{3}$ & 45 & $4.8^{7}$ & 100 \\
\hline 7.4 & 100 & 2.2 & 25 & $2.5^{*}$ & 46 & $11.3^{8}$ & 74 \\
\hline 7.5 & 100 & 2.6 & 34 & $3.3^{4}$ & 60 & & \\
\hline 9.1 & 100 & 3.2 & 0 & 4.0 & 21 & & \\
\hline $10.0^{1}$ & 100 & $5.0^{2}$ & 16 & $4.3^{*}$ & 35 & & \\
\hline \multirow[t]{7}{*}{15.0} & 100 & 5.1 & 33 & 5.9 & 41 & & \\
\hline & & 16.5 & 14 & $6.4^{*}$ & 62 & & \\
\hline & & & & 6.7 & 73 & & \\
\hline & & & & 7.0 & 45 & & \\
\hline & & & & $9.0^{*}$ & 51 & & \\
\hline & & & & $9.3^{5 *}$ & 79 & & \\
\hline & & & & $16.2^{*}$ & 60 & & \\
\hline
\end{tabular}

Abbreviations: PI, potentiation index; \% $\mathrm{Tt}$, percentage transient tension; $\mathrm{T}$, testosterone.

Superscripts: 1, male represented in Fig. 1; 2, female represented in Fig. 1; 3, testosterone pellet implanted for a month; pellet removed 5 wccks prior to tcsting; 4 , testosterone pellet implanted for 3 months and removed a year prior to testing; 5, female represented in Fig. 2; 6, castrated for 5 weeks; 7, castrated for 3 months; 8 , castrated for 3 weeks. Asterisk, gonadectomized.

$p<0.05$ for males vs females, PI and \% Tt, and for testosterone-treated females vs females, PI and \% Ti.

a male and a female isolated larynx at 2 stimulus rates. At slower stimulus rates $(29 \mathrm{~Hz})$, both male and female laryngeal muscle produce compound action potentials that potentiate throughout the stimulus train. Each compound action potential elicits a discrete tension transient (Fig. 1, $A, B$ ). The fastest rate at which female laryngeal muscle can produce discrete tension transients in response to nerve stimulation varies from 17 to $29 \mathrm{~Hz}$. Nerve stimulation at more rapid stimulus rates $(66 \mathrm{~Hz}$, a rate mimicking the fast portion of the mate call) results in compound action potential potentiation and discrete tension transients only in males (Fig. $1 C$, Table 1). Male laryngeal muscle is capable of producing discrete tension transients in response to stimulus rates up to $100 \mathrm{~Hz}$ (data not shown). In response to the same stimulus rate $(66 \mathrm{~Hz})$, female laryngeal muscle produces significantly less compound action potential potentiation than males. All of the potentiation occurs at the beginning of the train, the compound action potential rapidly attaining its maximum value. No discrete tension transients (Fig. 1D, Table 1) are produced; instead, a maintained tension with smaller transients superimposed is observed (Fig. 1D).

In summary, male laryngeal muscle is capable of generating strong discrete tension transients in response to rates of stimulation greatcr than those rcquircd for producing the matc call. Female laryngeal muscle produces no discrete tension transients in response to nerve stimulation at this rate.

\section{Effect of androgen on physiological properties of the larynx}

Castrated males do not mate call (Wetzel and Kelley, 1983). Treatment of adult females with androgen results in the production of poorly modulated trills in which the click frequency is faster than that of typical ticking (Hannigan and Kelley, 1986). The physiological differences between male and female larynges described above suggested that androgen-induced changes in the larynx might account for concomitant changes in behavior. Physiological responses to nerve stimulation were examined in larynges from androgen-treated females and castrated males.
Compound action potential potentiation and tension records from castrated males are similar to those of intact males (Table 1). Castrated males continue to exhibit discrete tension transients in response to rapid rates of nerve stimulation, even when tested 3 months after castration. Androgen treatment of adult females partially masculinizes neurally evoked responses of laryngeal muscle. The most rapid rate of nerve stimulation at which discrete tension transients can be produced is increased (range for intact females, $17-29 \mathrm{~Hz}$; range for androgen-treated females, $25-50 \mathrm{~Hz}$ ). The percentage transient tension observed at the fast mate-call stimulus rate is significantly increased (Table 1). The potentiation index observed in response to nerve stimulation at the fast mate-call rate also increases significantly (Table 1). However, tension records from androgen-treated females are never completely masculinized. At mate-call stimulus rates, some maintained tension is always present (Fig. 2, taken from one of the most masculinized females; data from all females, Table 1). No significant difference in PI or percentage transient tension was found between gonadectomized and unoperated androgen-treated females.

In 2 animals, the androgen pellet was removed prior to testing (5 weeks and 1 year). Responses of laryngeal muscle to nerve stimulation in thesc animals rctained their masculine features (Table 1). These results suggest that short-term androgen treatment is sufficient to effect long-term alterations in laryngeal physiology.

\section{Sound production by the isolated larynx}

The data presented thus far indicate that, in response to nerve stimulation, male laryngeal muscle is capable of producing tension transients at rates equal to or greater than those required for the fast trills of mate calling, while female laryngeal muscle cannot produce discrete tension transients at these rates. To relate sex differences in production of tension transients to observed behavioral dimorphisms, the necessary conditions for click production were determined. If a transient tension super- 

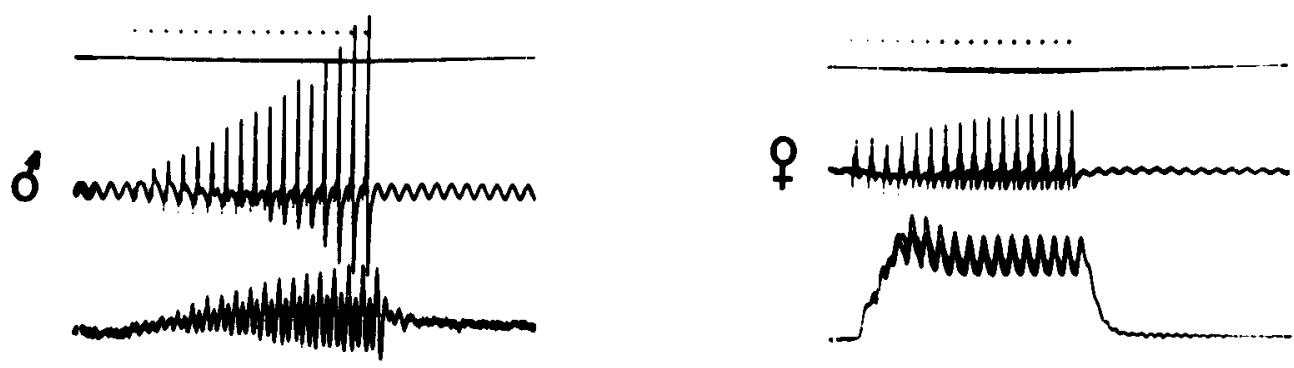

Figure 2. Responses of male, female, and testosterone-treated female larynges to stimulus trains mimicking the fast portion of the mate call. The treated animal was gonadectomized and received a testosterone $(T)$ pellet for a month. Traces as described in Figure 1. Stimulus train was $66 \mathrm{~Hz}, 250 \mathrm{msec}$ duration. Calibration bar: vertical compound action potential $=5 \mathrm{mV}$ (male), $10 \mathrm{mV}$ (female and T-treated female), tension $=3.5 \mathrm{gm}$; horizontal, 50 msec.

imposed on maintained tension is sufficient for the production of a click, the female larynx should be capable of producing rapid clicks. If, however, only discrete tension transients result in a click, females should be incapable of sound production. This point is particularly important when considering the results for androgen-treated females, the tension records of which at rapid rates of nerve stimulation indicate a significant increase in the amplitude of tension transients.

Tension transients and compound action potentials were recorded as described above. In addition, both laryngeal nerves were stimulated simultaneously and a small microphone was placed above the arytenoid disks to record sound production. Sounds produced by the isolated larynx were spectrographically analyzed to insure that these were true clicks, i.e., had spectral properties similar to those produced by an intact animal.

Figure 3 compares the sounds produced by an isolated male larynx with those of an intact male (data from Wetzel and Kelley, 1983). In both cases, individual clicks are composed of multiple oscillations that decrease in amplitude with time (Fig. $3 A$ ). The isolated larynx and calling male both contain a dominant frequency peak at $1.8 \mathrm{~Hz}$ (Fig. 3, B,C). A second peak at $2.3 \mathrm{~Hz}$ (isolated larynx) and $2.0 \mathrm{~Hz}$ (intact animal) is also present. In addition, the isolated larynx produces a higher frequency (3.4 $\mathrm{Hz}$ ) not present in the intact animal (Fig. $3 \mathrm{C}$ ). Thus, the isolated larynx is capable of producing sounds with similar spectral properties to sounds produced by intact animals.

The question of how tension relates to the generation of a click can now be addressed. In the isolated male larynx, a click is produced in response to each tension transient above a critical amplitude (Fig. $4 A$ ). At the initiation of the stimulus train, tension transients are small, and no click (top trace) is generated. When the tension attains a threshold magnitude (following the third compound action potential), a click is faithfully produced in response to each subsequent tension transient throughout the stimulus train. Similar results have been obtained in response to $66 \mathrm{~Hz}$ stimulus trains (data not shown), although the amplitude of the click, as in intact animals, is attenuated.

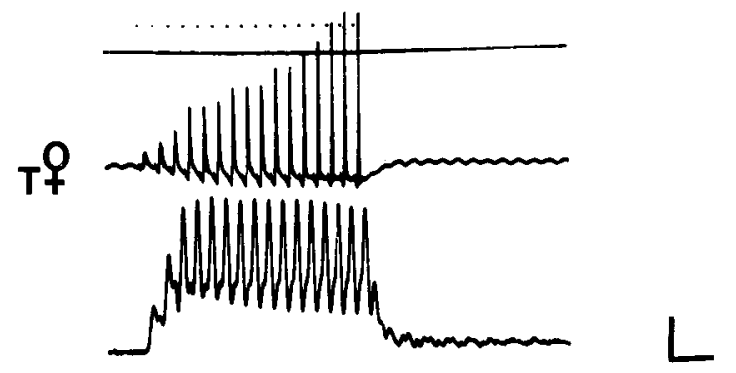

The relationship between tension and click production is the same in the female as it is in the male (Fig. 4). When the female larynx is stimulated at a rate $(17 \mathrm{~Hz})$ slow enough to permit production of discrete tension transients (Fig. 4C), a click is produced in response to each stimulus. However, when the stimulus rate $(33 \mathrm{~Hz})$ results in a maintained tension, only one click is produced near the beginning of the stimulus train (Fig. $4 B$ ). Presumably, maintained tension prevents click production by holding the arytenoid disks apart. In conclusion, only stimulus trains that allow discrete tension transients result in production of trains of clicks. Since the mechanism of click production is the same in males and females, the ability to generate calls at a given rate in response to nerve stimulation is solely dependent on the physiological properties of the larynx.

\section{Discussion}

Expression of a sexually dimorphic behavior requires the functioning of both central and peripheral elements. One role of sex hormones is to mediate the survival and/or competence of these elements in the appropriate sex. In rodents, behaviors necessary for successful fertilization are controlled by a group of muscles (levator ani/bulbocavernosus) innervated by motor neurons in the spinal nucleus of the bulbocavernosus (SNB; Breedlove and Arnold, 1980). Adult females entirely lack these muscles and possess significantly fewer SNB neurons than males (Breedlove and Arnold, 1981). In rats and mice, the presence of androgenic hormones early in male development maintains the penile musculature, which subsequently saves SNB motor neurons from ontogenetic cell death (Breedlove and Arnold, 1983a, b; Nordeen et al., 1985; Breedlove, 1986). Thus, females are prevented from expressing a male-specific behavior by loss of both the required muscles and their motor neurons. In the Japanese toad, both sexes have the neural and muscular elements necessary for generating the release call, which is produced only by males. Females are mute, due to an insufficient vocal box (Kusunoki et al., 1986). Limitations on female vocal production in birds have been attributed to sex differences in the differentiation of 


\section{Isolated Larynx}

A

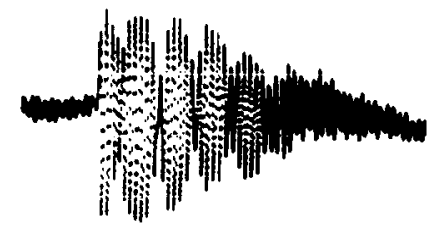

$(\mathrm{KHz})$

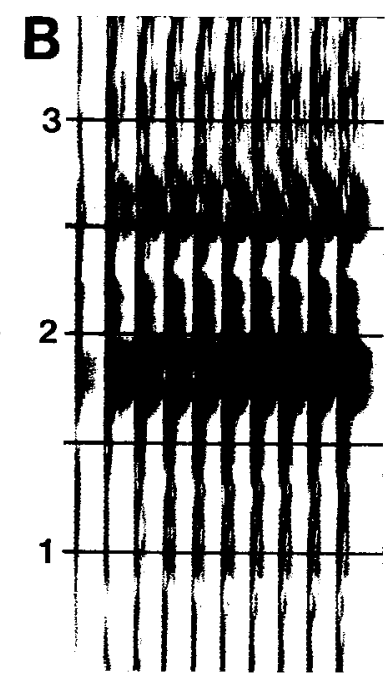

(dB)

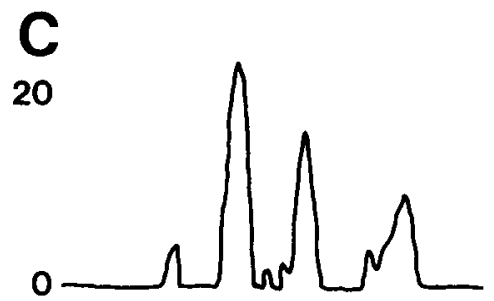

1

\section{Intact Animal}
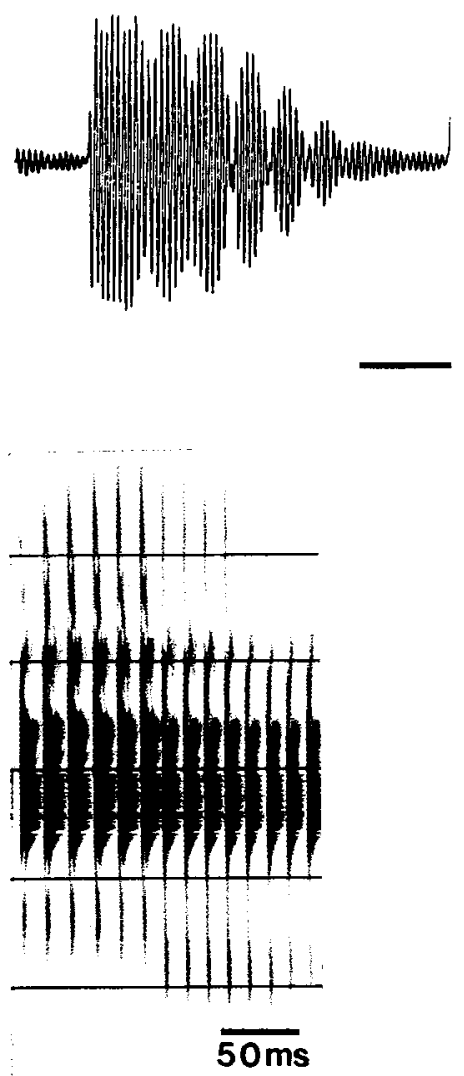

11111

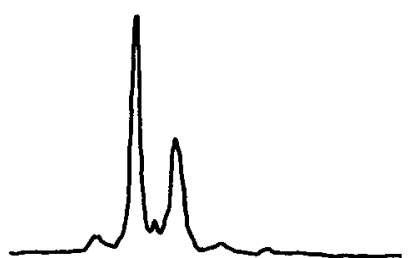

1 2 3 $(\mathrm{KHz})$
Figure 3. Spectral analysis of sounds produced by an isolated male larynx and an intact male frog. $A$, Oscillograph of an individual click. These records illustrate amplitude vs. time. Calibration bar, 5 msec. $B$, Sonograph of sounds produced by an isolated larynx in response to a $33 \mathrm{~Hz}, 250 \mathrm{msec}$ duration stimulus train and an intact male during the slow portion of the mate call. The dominant frequency $(1.8 \mathrm{kHz})$ is indicated by the thickness of the line. These records illustrate frequency vs time. $C$, Power spectrum of an individual click. These records illustrate amplitude vs frequency.

$(\mathrm{KHz})$

appropriate brain pathways, afferent to vocal motor neurons (Arnold, 1980; Gurney and Konishi, 1980; Konishi and Akutagawa, 1985). Both males and females possess a functional vocal organ, the syrinx. The syringcal muscles and motor neurons are not dimorphic in cell number (Bleisch, 1982; Arnold, personal communication). Vocal control nuclei in the bird telencephalon are masculinized by early hormone treatment; this treatment results in the production of male vocalizations (Gurney, 1982). Thus, in birds, CNS differences are held responsible for sex differences in vocal expression.

Vocal behavior in $X$. laevis is regulated in yet another fashion. Both sexes vocalize, but the calls are dimorphic in temporal properties. Females maintain the peripheral elements necessary for sound production, but these are not sufficiently developed to permit the rapid trilling that characterizes male mate calls. At metamorphosis, the larynx is sexually monomorphic and feminine in both sexes (Sassoon and Kelley, 1986). Androgeninduced myogenesis in the male results in a male larynx with 8 times the number of muscle fibers as in the female (Sassoon and Kelley, 1986; Sassoon et al., 1986). Muscle myosin ATPase and SDHase activity profiles are also controlled by male-specific patterns of hormone secretion (Sassoon et al., 1987).

Physiological properties of the isolated larynx correspond remarkably well to the vocal behavior of intact $X$. laevis. The male mate call is characterized by alternating fast and slow trill phases; our results indicate that these very rapid trills are readily produced by the discrete tension transients generated by male laryngeal muscles in response to nerve stimulation. The male larynx is capable of generating discrete tension transients up to $100 \mathrm{~Hz}$, rates exceeding the fast portion of the mate call. The female larynx cannot produce the rapid trills of a mate call. Females do use a simpler and much slower call, ticking, to 


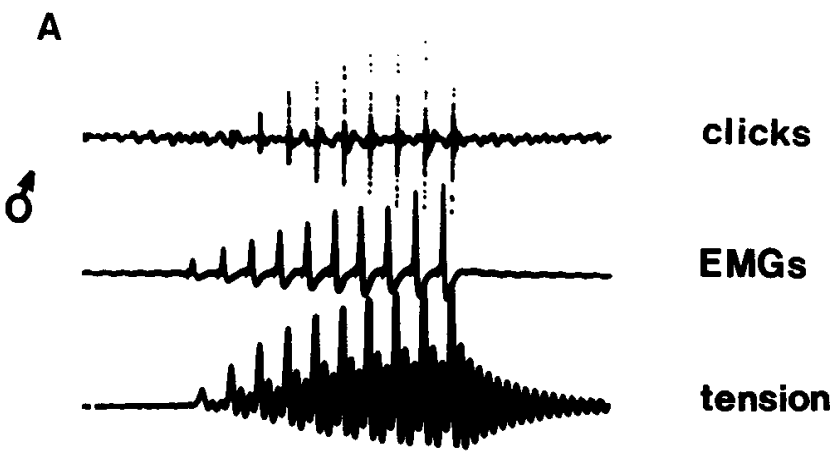

$33 \mathrm{~Hz}$

B

Figure 4. Relation between sound production and muscle response to nerve stimulation in male and female larynges. Top trace, microphone recording; middle and bottom traces as in Figure 1. Calibration bar: vertical, compound action potential $=5 \mathrm{mV}$; tension $=3.5 \mathrm{gm}$; horizontal, $50 \mathrm{msec}$.

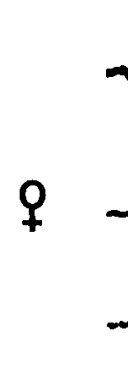

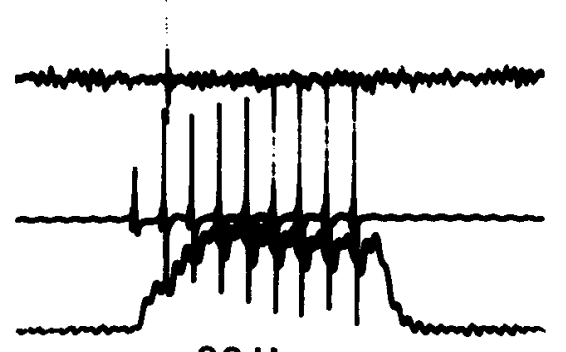

$33 \mathrm{~Hz}$
C

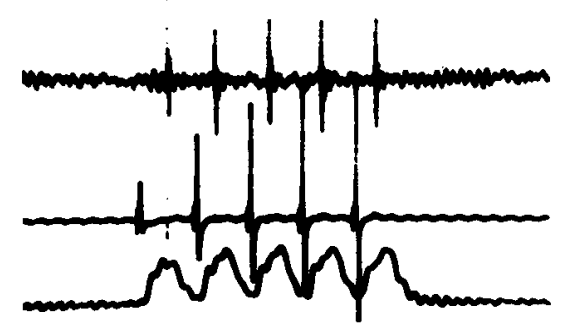

$17 \mathrm{~Hz}$ prevent or terminate clasping by males. The rate of click production in ticking varies from 6 to $17 \mathrm{~Hz}$; the higher rate characterizes release calls emitted during clasping (unpublished observations). The stimulus rate at which isolated female larynges can produce discrete tension transients ranges from 17 to 29 $\mathrm{Hz}$. Thus, ticking is readily produced by the less robust female larynx. Gonadectomized, androgen-treated adult females exhibit rapid ticking (mean rate, $50 \mathrm{~Hz}$; Hannigan and Kelley, 1986). This rate is the upper limit for discrete tension transient production displayed by the isolated larynges of androgen-treated females. Such females do not produce the rapid trills that characterize male typical mate calls (Hannigan and Kelley, 1986). Our results indicate that this behavioral characteristic is due, at least in part, to the inability of the female larynx to produce rapid discrete tension transients.

Scx-spccific vocalizations can be distinguished by the temporal pattern of trills. Since the muscle never contracts more than once in response to a single stimulus pulse to the nerve, we presume that the temporal pattern is controlled by events within the CNS. Since males exhibit both mate calling and ticking (for review, see Kelley, 1986), their nervous systems can generate either pattern of activity. Females also tick but do not mate-call (Hannigan and Kelley, 1986). We do not know whether the female CNS is capable of generating the mate-call pattern. The present results, however, show that even if the female CNS could produce the male vocal pattern, the periphery could not respond. Castrated males do not mate-call, despite the fact that isolated larynges from these animals are physiologically similar to intact males. Therefore, while a physiologically competent vocal organ is essential for masculine behavioral expression, the larynx must also be driven by the correct pattern of activity generated within the CNS.

Limitations on the rate of click production could be imposed centrally or peripherally. Females could be unable to mate-call solely because the laryngeal muscles do not receive the appro- priate activity from laryngeal motor neurons. If this were the case, male and female muscle should respond identically to similar stimulus trains presented to the laryngeal nerve. In fact, we observe the opposite result: identical stimuli produce different muscular responses in the 2 sexes. Therefore, the physiological characteristics of the larynx constrain the rate of click production.

The larynx produces sound by movement of the arytenoid disks, movement generated by muscle contractions induced by nerve activity. In principle, sex differences in the ability of the isolated larynx to generate sounds might be due to dimorphisms in the disks, in the muscles, and/or at the neuromuscular junction. Laryngeal cartilages are sexually dimorphic (Sassoon and Kelley, 1986). However, since both males and females call and since isolated larynges in both sexes produce clicks, the disks are capable of sound production in both sexes. Laryngeal muscle fiber type is also sexually dimorphic (Sassoon et al., 1987). Male laryngeal muscle is composed entirely of fast-twitch, fatigueresistant fibers, while female laryngeal muscle is composed predominantly of slow-twitch fibers. Transient (male) or maintained (female) tension produced by the isolated larynx in response to nerve activity probably reflects the twitch characteristics of the muscle. Sex differences in compound action potential potentiation reflect differences in muscle fiber recruitment. We suggest that the pronounced potentiation seen in males is due to successive recruitment of additional muscle fibers. Since all axons in the laryngeal nerve are excited during stimulation, the most likely mechanisms controlling fiber recruitment are temporal summation at the postsynaptic membrane or presynaptic facilitation. Intracellular recordings reveal that the majority of male laryngeal muscle fibers require facilitation to generate action potentials, while female laryngeal fibers do not (Tobias and Kelley, 1986). Thus, sex differences in fiber recruitment could result from presynaptic mechanisms.

In summary, then, we have described pronounced sex differ- 
ences in the larynx that set constraints on vocal expression. These differences appear to result from sexual dimorphism of both laryngeal muscle and its innervation. Sex differences in tcnsion production probably reflcct twitch and fatiguc propcrtics of laryngeal muscle fibers, while sex differences in compound action potential potentiation probably reflect events occurring at the nerve terminal.

\section{References}

Arnold, A. P. (1980) Effects of androgen on the volumes of sexually dimorphic brain regions in the zebra finch. Brain Res. 185: 441-444.

Bleisch, W. V. (1982) Neuromuscular junctions in androgen sensitive muscles: a model for synaptic plasticity. Ph.D. dissertation, Rockefeller University, New York, New York.

Breedlove, S. M. (1986) Cellular analysis of hormone influence on motoneural development and function. J. Neurobiol. 17: 157-176.

Breedlove, S. M., and A. P. Arnold (1980) Hormone accumulation in a sexually dimorphic motor nucleus in the rat spinal cord. Science 210: 564-566.

Breedlove, S. M., and A. P. Arnold (1981) Sexually dimorphic motor nucleus in the rat lumbar spinal cord: Response to adult hormone manipulation, absence in androgen-insensitive rats. Brain Res. 225. 297-307.

Breedlove, S. M., and A. P. Arnold (1983a) Hormonal control of a developing neuromuscular system: I. Complete demasculinization of the spinal nucleus of the bulbocavernosus in male rats using the antiandrogen, flutamide. J. Neurosci. 3: 417-423.

Breedlove, S. M., and A. P. Arnold (1983b) IIormonal control of a developing neuromuscular system: II. Sensitive periods for the androgen induced masculinization of the rat spinal nucleus of the bulbocavernosus. J. Neurosci. 3: 424-432.

Goy, R. W., and B. McEwen (1980) Sexual Differentiation of the Brain, MIT Press, Cambridge, MA.

Gurney, M. E. (1982) Behavioral correlates of sexual differentiation in the zebra finch song system. Brain Res. 231: 153-172.

Gurney, M. E., and M. Konishi (1980) Hormone induced sexual differentiation of brain and behavior in zebra finches. Science 208: 1380 1382.

Hannigan, P., and D. B. Kelley (1986) Androgen-induced alterations in vocalizations of female Xenopus laevis: Modifiability and constraints. J. Comp. Physiol. A 158: 517-527.

Kelley, D. B. (1980) Auditory and vocal nuclei in the frog brain concentrate sex hormones. Science 207: 553-555.

Kelley, D. B. (1981) Locations of androgen-concentrating cells in the brain of Xenopus laevis: Autoradiography with ${ }^{3} \mathrm{H}$-dihydrotestosterone. J. Comp. Neurol. 199: 221-231.

Kelley, D. B. (1982) Female sex behaviors in the South African clawed frog Xenopus laevis: Gonadotropin-releasing, gonadotropic and steroid hormones. Horm. Behav. 16: 158-174.

Kelley, D. B. (1986) Neuroeffectors for vocalization in Xenopus laevis: Hormonal regulation of sexual dimorphism. J. Neurobiol. 17:231248.

Kelley, D. B., J. I. Morrell, and D. W. Pfaff (1975) Autoradiographic localization of hormone-concentrating cells in the brain of an amphibian Xenopus laevis. I. Testosterone. J. Comp. Neurol. 164: 4762.

Konishi, M., and E. Akutagawa (1985) Neuronal growth, atrophy, and death in a sexually dimorphic song nucleus in the zebra finch brain. Nature 315: 145-147.

Kusunoki, M., M. Satou, and K. Ueda (1986) An electromyographic analysis of calling behavior in the Japanese toad, Bufo japonicus. Zool. Sci. 3: 395-399.

Nordeen, E., K. Nordeen, D. Sengelaub, and A. Arnold (1985) Androgens prevent normally occurring cell death in a sexually dimorphic spinal nucleus. Science 229: 671-673.

Picker, M. D. (1983) Hormonal induction of the aquatic phonotactic response of Xenopus. Behaviour 84: 74-90.

Ridewood, W. G. (1898) On the structure and development of the hyobranchial skeleton and larynx in Xenopus and Pipa; with remarks on the affinities of the aglossa. Linn. Soc. J. Zool. 26: 53-127.

Sassoon, D., and D. B. Kelley (1986) The sexually dimorphic larynx of Xenopus laevis: Development and androgen regulation. Am. J. Anat. 177: 457-472.

Sassoon, D., N. Segil, and D. Kelley (1986) Androgen-induced myogenesis and chondrogenesis in the larynx of Xenopus laevis. Dev. Biol. 113: 135-140.

Sassoon, D., G. Gray, and D. Kelley (1987) Androgen regulation of muscle fiber type in the sexually dimorphic larynx of Xenopus laevis. J. Neurosci. 7: 3198-3206.

Schmidt, R. (1976) Neural correlates of frog calling: Isolated brain stem. J. Comp. Physiol. 108: 99-113.

Schmidt, R. (1983) Neural correlates of frog calling: Masculinization by androgens. IIorm. Behav. 17: 94-102.

Schmidt, R. (1984) Neural correlates of frog calling: Preoptic area trigger of "mating calling." J. Comp. Physiol. 154: 847-853.

Segil, N., L. Silverman, and D. Kelley (1987) Androgen binding in a sexually dimorphic muscle of Xenopus laevis. Gen. Comp. Endocrinol. 66: 95-101.

Simpson, H. B., M. L. Tobias, and D. B. Kelley (1986) Origin and identification of fibers in the cranial nerve IX-X complex of Xenopus laevis: Lucifer yellow backfills in vitro. J. Comp. Neurol. 244: 430444.

Snedecor, G. W., and W. G. Cochran (1967) Statistical Methods, 6th ed., Iowa State University Press, Ames.

Tobias, M., and D. Kelley (1986) Dye coupling and physiology are sex specific properties of laryngeal muscle fibers in the frog Xenopus laevis. Soc. Neurosci. Abstr. 12: 1213.

Weintraub, A., D. B. Kelley, and R. Bockman (1985) Prostaglandin $\mathrm{E}_{2}$ induces receptive behaviors in female Xenopus laevis. Horm. Behav. 19: 386-399.

Wetzel, D. M., and D. B. Kelley (1983) Androgen and gonadotropin effects on male mate calls in South African clawed frogs, Xenopus laevis. Horm. Behav. 17: 388-404.

Wetzel, D. M., U. Haerter, and D. B. Kelley (1985) A proposed neural pathway for vocalization in South African clawed frogs, Xenopus laevis. J. Comp. Physiol. A 157: 749-761.

Yaeger, D. (1982) A novel mechanism for underwater sound production in Xenopus borealis. Am. Zool. 122: 887. 